\title{
Studies in biomimetic chemistry
}

\author{
Ronald Breslow \\ Department of Chemistry, Columbia University, New York NY \\ 10027, USA
}

Abstract: Mimics of DNA, of antibodies, and of enzymes are described.

\section{AN ISOMER OF DNA}

In Nature, DNA is based on 2'-deoxyribose and has its phosphate links between carbons $3^{\prime}$ and $5^{\prime}$ of adjacent nucleosides. However, the biochemical process by which the 2 '-OH group is removed could have occurred as readily to remove the 3 '-OH group, except for the enzyme selectivity that has evolved. Furthermore, one can make a case that 3'-deoxyribose could have arisen more easily under primitive earth conditions. For instance, the formation of ribose by the formose reaction (ref. 1) could have been followed by conjugate elimination to form the enol of the 2-ketone whose reduction would have led to 3'-deoxyribose. Why has Nature selected the 2'-deoxyribose derivatives for genetic building blocks?

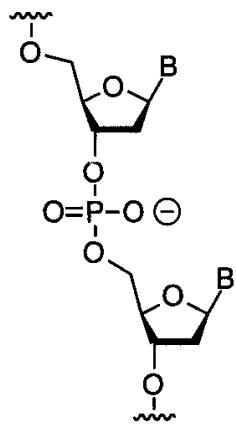

Normal DNA based on 2'-deoxyribose

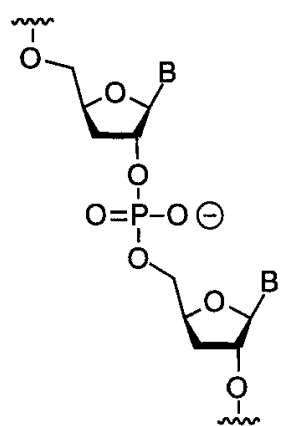

Our DNA isomer based on $3^{\prime}$-deoxyribose

In the course of other work we (ref. 2-4) have prepared the appropriate 3 'deoxyribose derivatives for incorporation in automatic DNA synthesis, and have prepared DNA oligomers (generally 16-mers) to study their properties. We have also incorporated the 3'-deoxyribonucleosides into DNA sequences containing the normal 2'-deoxyribonucleosides. The results are quite striking.

We find that a mixed sequence of $A^{*}$ and $T^{*}$ (the asterisk refers to the $3^{\prime}$-deoxy isomers of normal nucleosides) does not form a stable duplex with its complementary $\mathrm{T}^{*}$ and $\mathrm{A}^{*}$ strand under conditions in which the same sequences containing $A$ and $T$ form stable dimers. However, a 16-mer made up of $A^{*}$ units does form a complex with the 16 -mer of $T^{*}$ units, but only a triple helix. Since 
the triple helix requires two pyrimidines and one purine in each base interaction, it is formed with homopolymers that can bind two pyrimidine strands to one purine strand, but not with mixed sequences that do not have long runs of purines and, in their complements, of pyrimidines.

Our computer molecular modelling studies show that in normal DNA the double helix permits complete stacking overlap of the base residues, and this stacking is the feature that stabilizes DNA, by removing the aromatic residues from water and permitting them to be "solvated" by other polarizable organic residues. The hydrogen-bonding also contributes, but the hydrophobic effect from base stacking away from water is quite important, and DNA will dissociate in the presence of antihydrophobic agents such as urea or alcohols. Our molecular modelling reveals that with the 3'-deoxy isoDNA the stacking is less good, and significant areas of the bases are left exposed. The third strand helps to cover the exposed areas.

From these results, it is clear that 3'-deoxyribose would not have been a good basis for nucleosides to carry genetic information. A stable duplex in DNA with mixed sequences is required, and 3'-deoxyribose does not form stable duplexes. If some early organism tried this, it has long since failed to survive.

Our isoDNA also binds extremely poorly if at all to normal DNA, and even one isoDNA unit in a normal DNA strand seriously diminishes the affinity of that strand for its complement. However, we find that our isoDNA will form duplexes with normal RNA that are almost as stable as those of normal DNA with RNA. This finding indicates that we have an unusual tool, one that can recognize RNA sequences while ignoring the same sequences in DNA. This difference apparently reflects the well-known fact that DNA-RNA duplexes adopt a different helical structure than is common for most DNA dimers. We are currently doing the molecular modelling needed to account for these findings.

The isoDNA based on 3'-deoxyribose may even have antisense potential. We find that it is stable to mammalian nucleases that are a problem with normal DNA, so it or some analogs could survive and serve to bind RNAs with selectivity.

\section{CYCLODEXTRIN DIMERS AS ANTIBODY MIMICS}

In a number of studies (ref. 5-7) we have seen that two cyclodextrin rings linked to form dimers can have strong binding properties. Even with a single linkage joining two beta-cyclodextrin units we can bind appropriate substrates in water with binding constants up to $10^{9} \mathrm{M}^{-1}$, while with two links to form a hinge between the cyclodextrins and minimize rotation we see one binding constant exceeding $10^{11} \mathrm{M}^{-1}$. Such numbers, driven by the hydrophobic effect and very much dependent on a precise fit of the substrate into the cyclodextrin dimer so as to use both cyclodextrin cavities, put these materials in the class of antibodies.

The binding of various substrates tailored to fit these dimers is perhaps less interesting than the binding of natural materials such as steroids. Since the depth of a cyclodextrin cavity is ca. $7.8 \AA$, while cholesterol is ca. $15 \AA$ long in its extended conformation, a cyclodextrin dimer seemed a good bet for selective cholesterol binding if a very short linker was used between the two cyclodextrin units. Thus we prepared the dimer with a single sulfur atom between the two 
cyclodextrins linked to their primary faces, and saw that it was quite a good binder of cholesterol in water solution (ref. 7). It is apparently the best binder known, except for natural enzymes or antibodies.
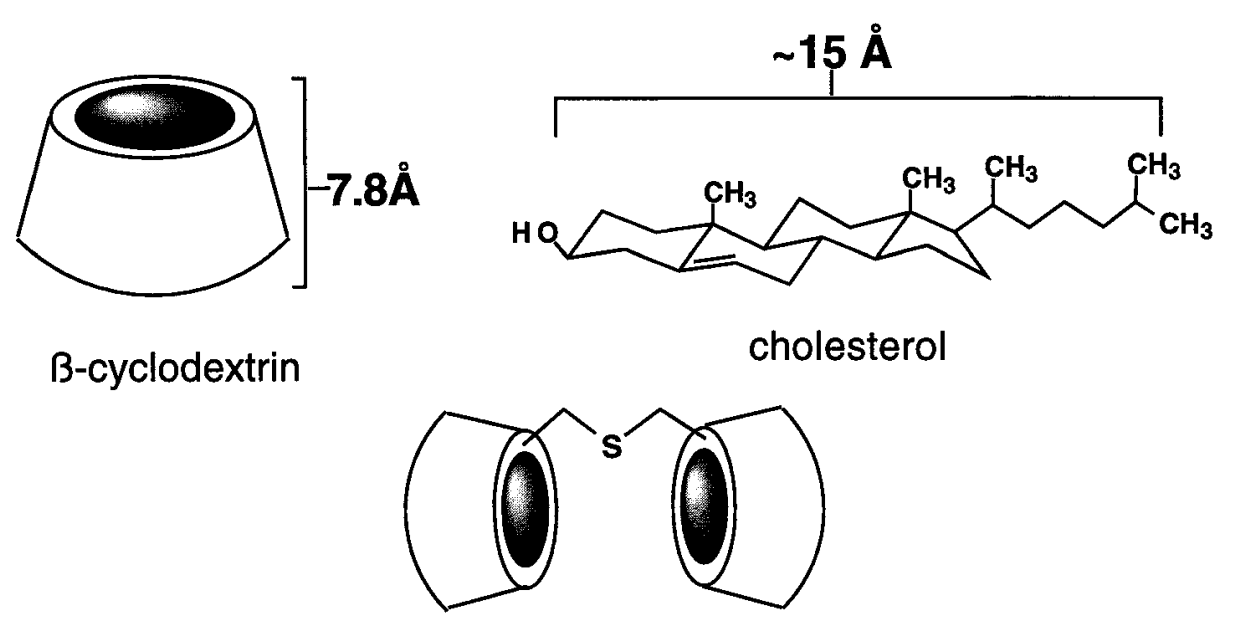

\section{ARTIFICIAL ENZYMES}

One of our major efforts over the years has been to prepare artificial enzymes that can mimic and extend the most exciting aspects of enzyme chemistry-the very large rate accelerations and very high selectivities in water solution, using hydrophobic binding of substrates into the catalyst. A recent review summarizes much of what we did in the past (ref. 8).

Enzymes generally combine a binding site with two or more catalytic groups. Using two catalytic groups, they often carry out in one simultaneous step processes that in simple solution would require two steps, with an unstable intermediate. This is the advantage to an enzyme of binding the substrate and two catalytic groups together, so such simultaneous processes are possible.

As one example, the enzyme ribonuclease A uses two imidazole groups, of His-12 and His-119, to perform the hydrolysis of RNA and of a cyclic phosphate derived from RNA. The $\mathrm{pH}$ vs. rate profile indicates that the two imidazole groups act with one as a base and the other, protonated, as an acid. Imidazole groups are particularly suited to this, since with a pK near neutrality they are the strongest bases that can exist unprotonated in neutral solution, while their protonated forms are the srongest acids that can exist without losing a proton. From isotopic studies used to carry out what is called a proton inventory it has been shown that the enzyme performs two proton transfers at once, taking up a proton with the basic imidazole at the same time the imidazolium ion donates a proton.

We have prepared several cyclodextrin bis-imidazoles and studied their ability to catalyze the hydrolysis of a bound cyclic phosphate ester, analogous to the process carried out by the enzyme (ref. 9-11). Mechanistic studies (12) suggested that the best catalyst would have the imidazoles on adjacent glucose residues, and that is what we found. Furthermore, proton inventory studies using hydrogen isotopes indicated that two protons move simultaneously in the hydrolysis reaction. In 
fact, the details of these isotope effects and of the $\mathrm{pH}$ vs. rate profile are remarkably similar to those of the enzyme.

In very recent work (ref. 11) we examined the selectivity and rate parameters for this process as a function of the fit of substrate and artificial enzyme, using three different cyclodextrins and two substrates. We saw that the binding constant and the rate constant were best for the combination of catalyst and substrate that fit really well, but the most striking effect was seen in the products. With most combinations the hydrolysis produced both products shown below, but in the ideal case with a rigid substrate locked tightly into the catalyst, as judged from computer models, only a single product could be detected. This system thus imitates many of the most important features of real enzymes.

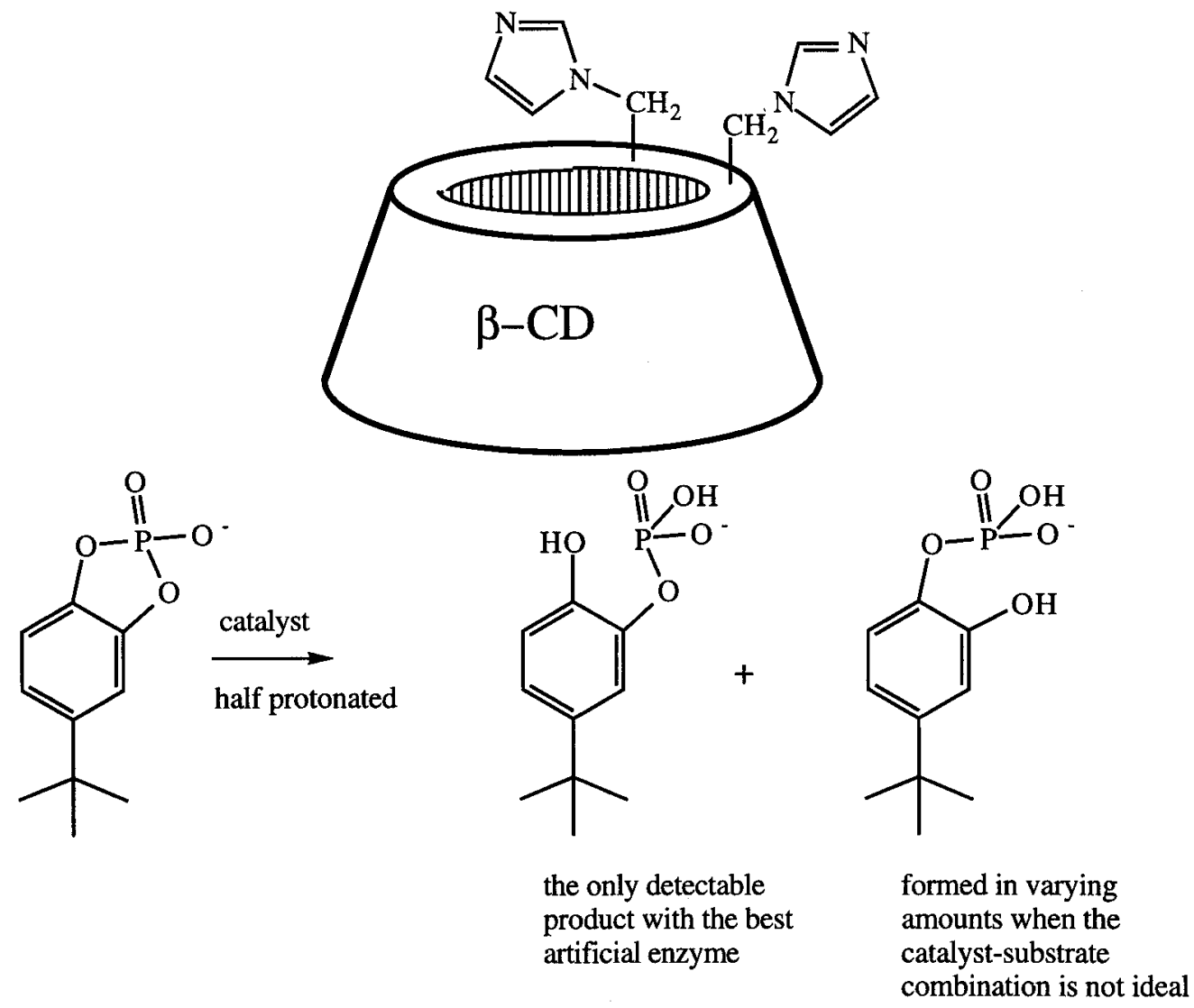

\section{REFERENCES}

1. R. Breslow, Tet. Letters, 21, 22 (1959).

2. R. Jin. W. H. Chapman, A. R. Srinivasan, W. K. Olson, R. Breslow, and K. Breslauer, Proc. Natl. Acad. Sci. USA, 90, 10568-10572 (1993).

3. T. L. Sheppard, A. T. Rosenblatt, and R. Breslow, J. Org. Chem. 59, 7243-7248 (1994).

4. T. L. Sheppard and R. Breslow, J. Am. Chem. Soc. 118, in press (1996).

5. R. Breslow, N. Greenspoon, T. Guo, and R. Zarzycki, J. Am. Chem. Soc. 111, 8296-7 (1989).

6. R. Breslow, S. Halfon, and B. Zhang, Tetrahedron, 51, 377-388 (1995).

7. R. Breslow and B. Zhang, J. Am. Chem. Soc. 118, 8495-6 (1996) and references.

8. R. Breslow, Accts. Chem. Res. 28, 146-153 (1995).

9. E. Anslyn and R. Breslow, J. Am. Chem. Soc. 111, 5972-3 (1989)

10. E. Anslyn and R. Breslow, J. Am. Chem. Soc. 111, 8931-2 (1989).

11. R. Breslow and C. Schmuck, J. Am. Chem. Soc. 118, 6601-5 (1996) and references.

12. R. Breslow, S. D. Dong, Y. Webb, and R. Xu, J. Am. Chem. Soc. 118, 6588-6600 (1996). 